\title{
GOMCL: a toolkit to cluster, evaluate, and extract non-redundant associations of Gene Ontology-based functions
}

\author{
Guannan Wang, Dong-Ha Oh and Maheshi Dassanayake ${ }^{*}$ (i)
}

\begin{abstract}
Background: Functional enrichment of genes and pathways based on Gene Ontology (GO) has been widely used to describe the results of various -omics analyses. GO terms statistically overrepresented within a set of a large number of genes are typically used to describe the main functional attributes of the gene set. However, these lists of overrepresented GO terms are often too large and contains redundant overlapping GO terms hindering informative functional interpretations.
\end{abstract}

Results: We developed GOMCL to reduce redundancy and summarize lists of GO terms effectively and informatively. This lightweight python toolkit efficiently identifies clusters within a list of $\mathrm{GO}$ terms using the Markov Clustering (MCL) algorithm, based on the overlap of gene members between GO terms. GOMCL facilitates biological interpretation of a large number of $\mathrm{GO}$ terms by condensing them into $\mathrm{GO}$ clusters representing non-overlapping functional themes. It enables visualizing GO clusters as a heatmap, networks based on either overlap of members or hierarchy among GO terms, and tables with depth and cluster information for each GO term. Each GO cluster generated by GOMCL can be evaluated and further divided into non-overlapping sub-clusters using the GOMCL-sub module. The outputs from both GOMCL and GOMCL-sub can be imported to Cytoscape for additional visualization effects.

Conclusions: $\mathrm{GOMCL}$ is a convenient toolkit to cluster, evaluate, and extract non-redundant associations of Gene Ontology-based functions. GOMCL helps researchers to reduce time spent on manual curation of large lists of GO terms, minimize biases introduced by redundant $\mathrm{GO}$ terms in data interpretation, and batch processing of multiple GO enrichment datasets. A user guide, a test dataset, and the source code of GOMCL are available at https://github.com/ Guannan-Wang/GOMCL and www.Isugenomics.org.

Keywords: Gene ontology clustering, GO annotations, Functional networks, GO similarity, Markov clustering, Functional genomics, High throughput omics

\section{Background}

High-throughput "omics" approaches are frequently employed to investigate expression changes and regulation of genes at a genome-wide level. Use of these genomic data often results in the identification of large lists of genes of interest. A standard approach

\footnotetext{
* Correspondence: maheshid@lsu.edu

Department of Biological Sciences, Louisiana State University, Baton Rouge, LA, USA
}

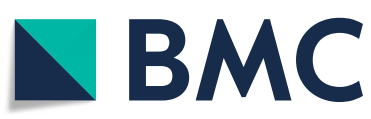

(c) The Author(s). 2020 Open Access This article is licensed under a Creative Commons Attribution 4.0 International License, which permits use, sharing, adaptation, distribution and reproduction in any medium or format, as long as you give appropriate credit to the original author(s) and the source, provide a link to the Creative Commons licence, and indicate if changes were made. The images or other third party material in this article are included in the article's Creative Commons licence, unless indicated otherwise in a credit line to the material. If material is not included in the article's Creative Commons licence and your intended use is not permitted by statutory regulation or exceeds the permitted use, you will need to obtain permission directly from the copyright holder. To view a copy of this licence, visit http://creativecommons.org/licenses/by/4.0/. The Creative Commons Public Domain Dedication waiver (http://creativecommons.org/publicdomain/zero/1.0/) applies to the data made available in this article, unless otherwise stated in a credit line to the data.

to summarize the functions of these genes is to determine the enriched functions represented by Gene Ontology (GO) terms and other functional associations extracted from databases such as KEGG [1-3], Reactome [4] and Pathway Commons [5], known as pathway enrichment analysis [6, 7]. This approach significantly simplifies the need from understanding the biological meaning embedded in individual genes in a large list, to the interpretation of enriched gene 
sets that could serve as a summary of enriched functions.

GO resources have become the most widely used knowledge base in terms of gene functions $[8,9]$, which provides a controlled hierarchy of GO vocabularies describing biological processes, molecular functions, and cellular components. However, this hierarchical functional annotation system presents a high level of redundancy as parent GO terms include or partially overlap with child GO terms and one gene could be annotated with seemingly unrelated GO terms. The computational tool, Enrichment Map [10, 11] was initially developed to overcome this problem by building a GO similarity network built on the overlap between gene sets annotated with each GO term. Yet, the identification of GO clusters within the GO similarity network in Enrichment Map does not define clusters and therefore the user has to separate groups based on a visual selection, which can be heavily affected by the layout of the network visualizations. As a result, when there are large numbers of similar GO terms, it is challenging to identify significant functional groups using Enrichment Map. Another comparable tool, ClueGO, identifies functional groups by first creating all possible initial groups with a user-defined number of GO terms showing similarities equal or above the predefined threshold and then iteratively comparing and merging these initial groups if the overlap between them is above the predefined threshold [12]. However, ClueGO can assign unique GO terms to multiple groups, making it challenging to identify nonredundant clusters [12]. Additionally, this tool does not accept direct output files from other commonly used GO enrichment tools such as BiNGO, g:Profiler, or agriGO. Both tools fall short at parallel processing a large number of distinct set of gene functions often encountered in large-scale -omics experiments. To address these limitations and generate a similarity-based functional GO network, we developed a new toolkit, GOMCL, that applies the Markov Clustering (MCL) algorithm [13-15] to identify cluster structures in GO networks in an unbiased approach. Each GO term is represented by a node and edges connect two GO terms that share a certain percentage of gene members in GOMCL. To further facilitate the interpretation of resulting functional groups, GOMCL allows users to generate hierarchy plots and provides subclustering options for any number of selected clusters. GOMCL is a user friendly python toolkit, which offers multiple visualization schemes and enables batch processing of large GO datasets to mine for functionally significant attributes.

\section{Implementation}

GOMCL is implemented in Python and allows grouping of lists of individual GO terms of interest into GO clusters using MCL (Fig. 1). GOMCL encapsulates its entire pipeline in a single command and offers default parameters with which users can expect optimal results.

\section{Input data}

The package accepts the direct outputs from a variety of commonly used GO enrichment analysis tools, including BiNGO [16], GOrilla [17], g:Profiler [18], and agriGO [19], as well as customized GO lists. Support for more enrichment tools will be provided. In addition to the GO lists, GOMCL requires a $\mathrm{GO}$ ontology file in $\mathrm{OBO}$ format from the Gene Ontology Consortium (http://geneontology.org/) as an input (Fig. 1).

\section{GOMCL workflow}

GOMCL first trims the input GO lists by removing overly broad GO terms whose size is greater than a user-defined threshold. For example, a large GO term such as biological regulation (GO:0065007) has over 12, 000 child GO terms, including 15,000 genes in Arabidopsis and is often uninformative as a term representing a meaningful biological function. GOMCL also enables users to separate input GO lists into biological process, molecular function, and cellular component categories or any combinations of these categories if clustering within different categories is preferred. Each term in the trimmed GO lists is then compared to each other, and similarity between any two GO terms is computed based on the overlaps between the members of these two GO terms as either a Jaccard Coefficient (JC) or an Overlap Coefficient (OC) [10]. Given any two GO terms, A and $\mathrm{B}$, the Jaccard Coefficient (JC) is calculated as $\mathrm{A} \cap \mathrm{B} / \mathrm{A} \cup \mathrm{B}$, and preferred for clustering of similarly sized GO terms. The Overlap Coefficient $(O C)$ is derived from $A \cap B / m i n$ $(\mathrm{A}, \mathrm{B})$, and works better to maximally reduce the redundancy between disproportionately sized GO terms. The construction of the GO term similarity network is initiated using only those interactions that pass a userdefined threshold for the Jaccard or Overlap coefficient of users' choice. MCL algorithm is subsequently applied to identify cluster structure in the initial network and assigns more similar GO terms into one cluster. The resulting GO clusters are ordered based on the number of genes in each cluster. GO terms with largest number of genes, or smallest enrichment $p$-value, or most other GO terms connected are selected and offered as potential representative GO terms for each cluster. GOMCL also reproduces the hierarchy of GO terms from the provided ontology structure for any user-selected clusters upon command to assist identification and interpretation of the functional themes of these clusters. A novel functionality enabled in GOMCL that is unavailable in previous tools for GO-network analysis, is the evaluation of clustering results by visualizing the distribution of similarity indexes between GO terms for each 


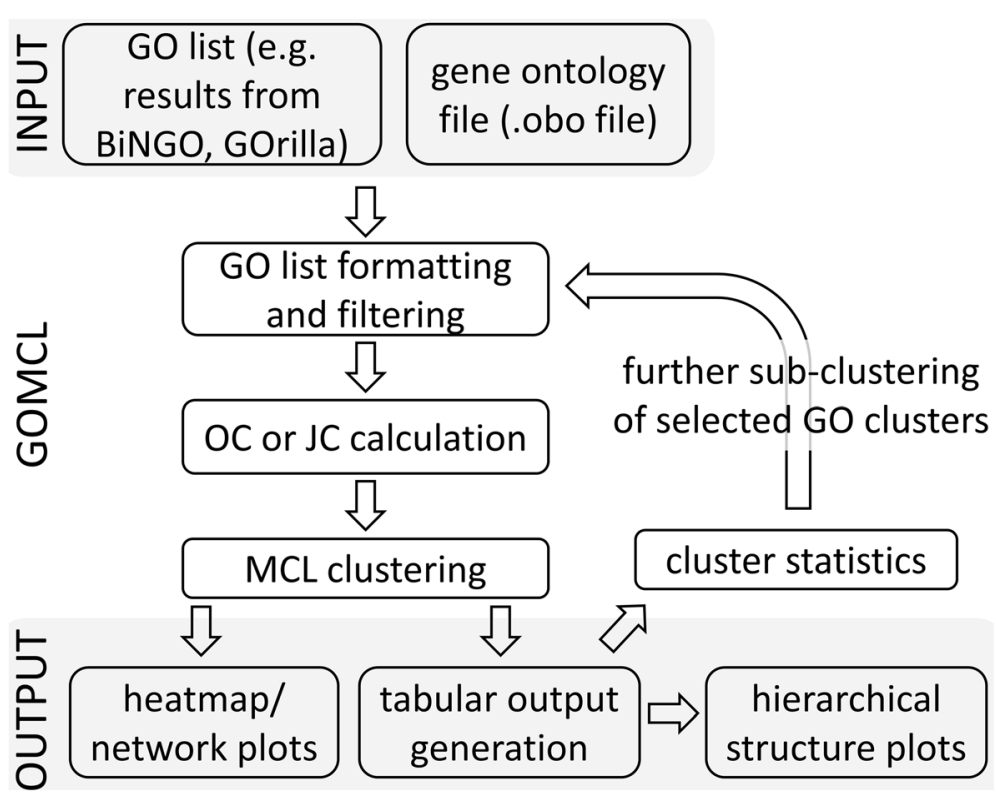

Fig. 1 The workflow of GOMCL clustering on GO enrichment test results

cluster. Taking this one step further, GOMCL includes a second module, called GOMCL-sub, which provides users customizable options to break down selected clusters produced by GOMCL into sub-groups with more specific functional themes. These functionalities combined, allows users to determine if there are distinct functional themes present in primary clusters and further identify these sub-structures in clusters of interest.

\section{Output format}

The standard GOMCL output consists of a heatmap (Fig. 2a), a graphical GO-similarity-network based on the clustering results (Fig. 2b), a tabulation of each GO term with cluster information and depth information [20] from the provided ontology structure, and a summary file for all clusters (Fig. 2c). Graphical presentations of similarity index distribution (Fig. 3) and GO hierarchy for individual clusters (Fig. 4) are generated if the user chooses that option to create additional result files. If the user plans to generate cluster depth information for each GO term and build GO hierarchies as an output file, we recommend that the same version of the GO ontology file used in the GO enrichment analysis tool where the GO input list is created to should be used as an input for GOMCL. In addition to the graphical outputs, the user can opt to generate simple interaction format files with either similarity between GO terms or GO hierarchy (Additional file 2), both of which can be directly used as inputs, together with the information about clustered GO terms (Additional file 1), to Cytoscape [21] for further manipulation of GO network visualization.

\section{Results}

As a proof of concept, we performed a GOMCL run on a list of over-represented GO terms identified from genes differentially expressed between two GFP tagged cell populations of Arabidopsis roots in a published study [22] to highlight the functional use of GOMCL. In this published study, a xylem-specific promoter was used to drive the expression of GFP in Arabidopsis, and root proximal meristem cells were later separated into two populations based on the intensity of GFP signals. Cells with high GFP signals were assumed to be close to the quiescent center while cells with low GFP signals were assumed to be located away from the quiescent center. A microarray analysis was then conducted to compare the two cell populations and the authors aimed to see a difference in gene expression associated with cell division between these two cell populations.

\section{Cluster identification}

We used GO terms that had less than 3500 genes annotated under each GO annotation for Arabidopsis, to allow identification of specific functional traits associated with the published study. This resulted in 244 total GO terms (out of 251) enriched in genes expressed higher in the cell population with high GFP intensity (Additional file 1). The default Overlap Coefficient of 0.5 and granularity of 1.5 were used for cluster identification. These cutoffs can be set by the user. Among the $244 \mathrm{GO}$ terms, GOMCL identified five distinct clusters with minimal overlap between clusters and extensive overlaps among GO terms within each cluster (Fig. 2a). The largest cluster (C1) included $124 \mathrm{GO}$ terms and was mainly related to 


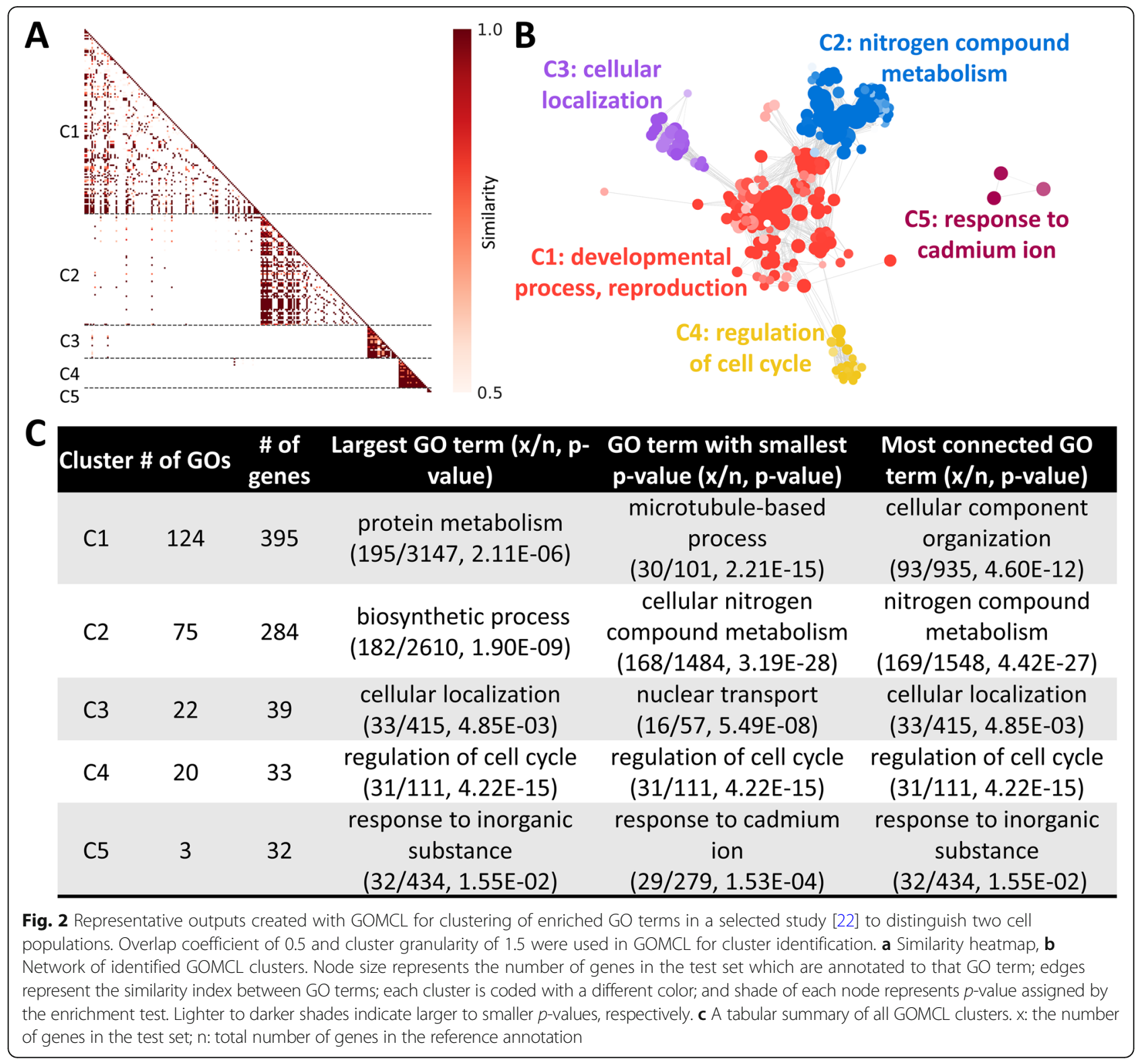

developmental processes and reproduction (Fig. 2b, c). The 4th largest cluster, albeit comprising only 33 genes from 20 GO terms (Fig. 2b, c), was overrepresented in genes associated with regulation of cell cycle and was also found to be mostly associated with the largest cluster. These representative functional groups and associations reflected the essential difference in the growth stages between the high GFP cells (cells assumed to be close to the quiescent center) and low GFP cells (cells located away from the quiescent center) in the targeted study where it aimed to see a difference in gene expression associated with cell division between these two cell populations [22] The reduction from over $200 \mathrm{GO}$ terms to $5 \mathrm{GO}$ clusters preserved the enriched functional themes and facilitated the explanation of major patterns identified among $\sim 1000$ differentially expressed genes.

\section{Cluster quality evaluation and sub-clustering process}

To demonstrate the use of cluster quality evaluation and sub-clustering, we first enabled options to generate similarity index distributions for all five clusters identified by GOMCL. As shown in Fig. 3, the majority of similarity indexes between $\mathrm{GO}$ terms within cluster $\mathrm{C} 3, \mathrm{C} 4$, and $\mathrm{C} 5$ were greater than 0.5 . However, there is a large proportion of $\mathrm{GO}$ terms from cluster $\mathrm{C} 1$ and $\mathrm{C} 2$ showing no or small overlaps with other GO terms from the same cluster. To assist determining whether cluster $\mathrm{C} 1$ and $\mathrm{C} 2$ should be further separated into groups with more specific 


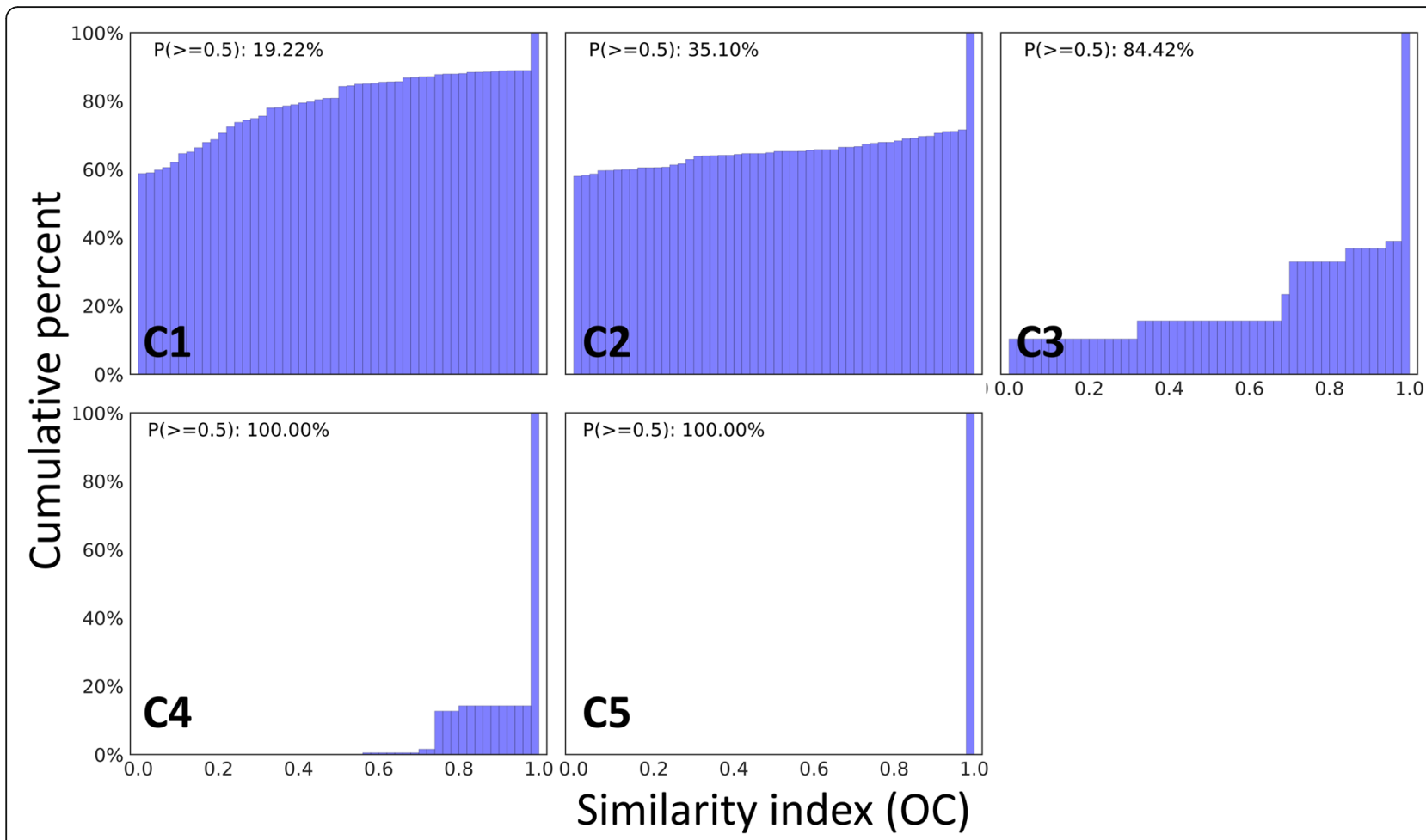

Fig. 3 Cumulative distribution of similarity indexes between GO terms within each GOMCL cluster identified from test data reported in Wendrich et al. 2017. $P(>=0.5)$ indicates the proportion of similarity indexes greater than 0.5 among all the similarity indexes within a given cluster

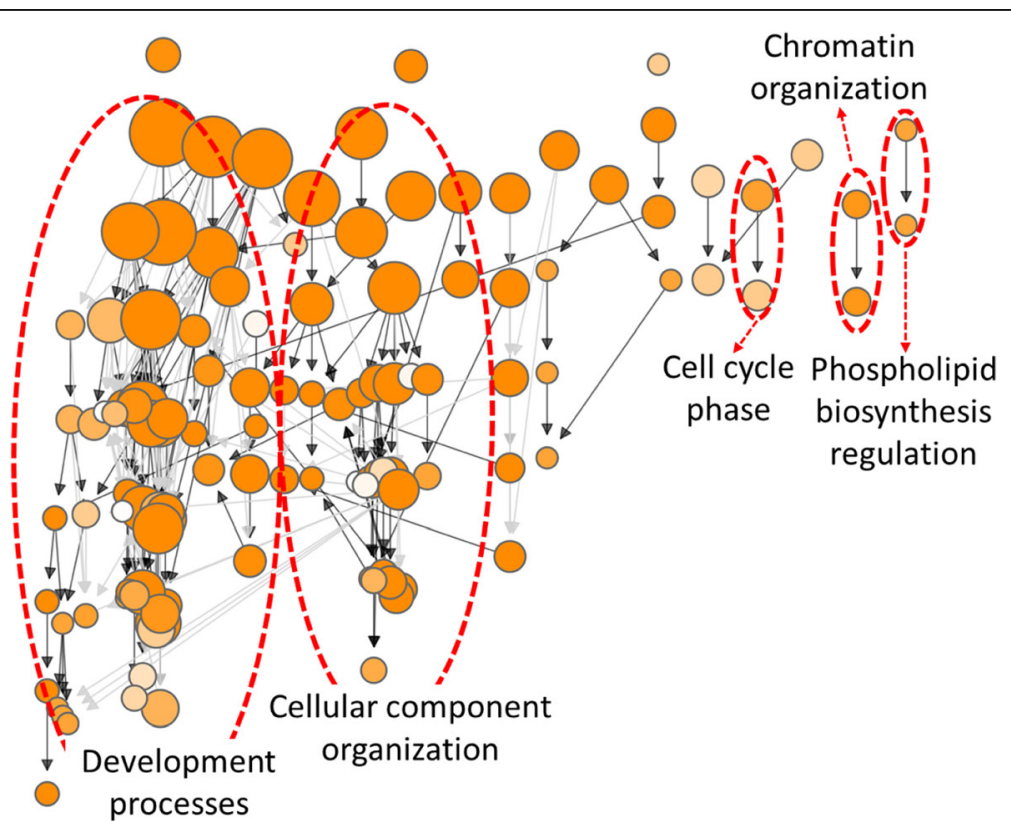

Fig. $4 \mathrm{GO}$ hierarchical structure produced using GOMCL for cluster C1 described in Fig. 1. Edges represent the parent/child relationships of the GO terms. The black edges connect parent and child terms that are directly linked, while the grey edges indicate connections with intermediate $\mathrm{GO}$ terms between the parent and child terms. Node size represents the number of genes in the test set which are annotated to that GO term; and shade of each node represents $p$-value assigned by the enrichment test. Lighter to darker shades indicate larger to smaller $p$-values, respectively. The main hierarchical branches are marked by red circles 
functional themes, we plotted GO hierarchies for these two clusters with GOMCL parameter -hg on (Fig. 4, Additional file 3). Several distinct branches were identified from the hierarchical structures of GO terms within each cluster (Fig. 4, Additional file 3), indicating the possible presence of distinct sub-groups within these clusters. We further employed GOMCL-sub, which was designed to analyze selected clusters produced by GOMCL, to identify the sub-groups within large clusters such as $\mathrm{C} 1$ and $\mathrm{C} 2$ when users need to identify more distinct and functionally informative sub-clusters. To increase clustering sensitivity, we reduced the cutoff of GO term size to 2000 and increased the granularity to 1.8 , and left similarity cutoff unchanged. With these parameters, GOMCL-sub passed 122 GO terms from cluster $\mathrm{C} 1$ and was able to separate them into 4 sub-groups (Fig. 5a). 117 out of the 122 GO terms were assigned to the two largest sub-groups $(\mathrm{C} 1-1$ and $\mathrm{C} 1-2)$, whose main functional themes were associated with development processes and cellular component organization, respectively (Additional file 4). These two sub-groups recapitulated and extended the main theme of the original cluster, $\mathrm{C} 1$. Additionally, this led to the identification of more informative details of the cluster. For instance, the development process associated with cluster $\mathrm{C} 1-1$ was mainly composed of GO terms involved in anatomical development processes (e.g. root development) (Fig. 5b, Additional file 5). Whereas, over $85 \%$ of the GO terms in cluster C1-2 were involved in processes such as cellular component organization that represented chromosome organization and cytoskeleton organization. More importantly, cell cycle processes were found to intersect with cellular component organization and seemed to link different processes assigned to the $\mathrm{C} 1-2$ sub cluster (Fig. 5c, Additional file 5). These results combined, provided a more detailed overview of how development processes and cell cycle regulation were different between the two cell populations in the target study.

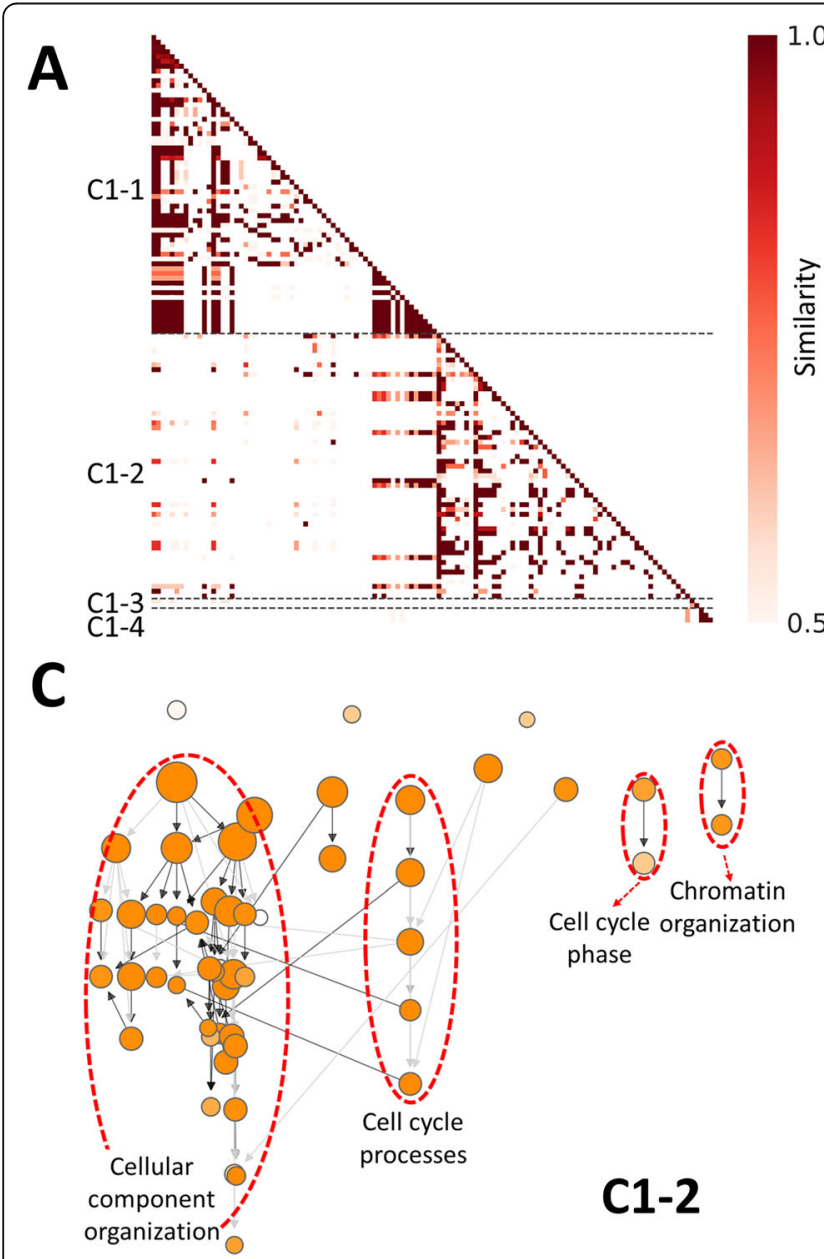

Fig. 5 Sub-clustering results produced by GOMCL-sub on cluster C1 described in Fig. 1. a Similarity heatmap of sub-groups identified by GOMCLsub. $\mathbf{b}$ and $\mathbf{c}$ GO hierarchical structures of $\mathrm{C} 1-1$ and $\mathrm{C} 1-2$ sub-clusters. The black edges connect parent and child terms that are directly linked, while the grey edges indicate connections with intermediate $\mathrm{GO}$ terms between the parent and child terms. Node size represents the number of genes in the test set which are annotated to that GO term; and shade of each node represents $p$-value assigned by the enrichment test. Lighter to darker shades indicate larger to smaller $p$-values, respectively. The main hierarchical branches are marked by red circles 
Contrasting to cluster $\mathrm{C} 1$, cluster $\mathrm{C} 2$ did not appear to contain additional sub-cluster structure that could be separated further, when GOMCL-sub was applied. The visualization of GO hierarchy of GOMCL clusters and further identification of sub-groups with more specific functional themes by GOMCL-sub greatly contribute to biological interpretations by facilitating objective clustering and extraction of overrepresented functional associations.

Notably, GOMCL identified these 5 clusters out of a list of $251 \mathrm{GO}$ terms and generated associated result files in $\sim 2$ min while using only $300 \mathrm{Mb}$ of RAM (easily found in most desktops/laptops). GOMCL-sub further separated cluster $\mathrm{C} 1$ and $\mathrm{C} 2$ clusters in less than 2 mins with similar amount of memory used. Given the efficiency of the toolkit, this can be easily implemented to conduct batch processing of multiple datasets associated with large -omics datasets.

While the proof of concept analyses described above using GOMCL is used to highlight functional associations drawn from a typical RNAseq experiment, the use of GOMCL is not limited to summarizing functional processes from RNAseq data. For example, it has been recently successfully used in summarizing gene functions associated with multiple epigenetic marks in rice under phosphorus starved conditions [23]. Additionally, GOMCL can be used to cluster and summarize biological processes associated with GO-slim ontologies [24], similar to its use with the standard GO terms. To further assess and highlight its versatility, we compared the features of GOMCL to several existing GO term clustering tools in Table 1.

\section{Discussion}

GOMCL is an open-source Python toolkit to identify clusters among GO term similarity networks using the MCL clustering algorithm. This toolkit allows grouping of GO terms into functional clusters to further simplify the interpretation of large datasets, reduce redundancy of functional interpretations, and especially when visual identification of cluster structure is not feasible due to a large number of enriched GO terms often found in -omics data (see Table 1 for a comparison with other available tools). To better evaluate and understand the resulting clusters, GOMCL offers options to visualize similarity indexes between GO terms and GO hierarchy. A second module, GOMCL-sub, is further introduced to further examine large clusters when users suspect that two or more distinct minimally overlapping functions might be captured in one large cluster. We demonstrated the use of GOMCL in successfully capturing the functional themes associated with a published study [22] as proof of concept of the toolkit. We showed that GOMCL built a concise and informative view of biological processes different between the two conditions tested in the target study and summarized the main differences. It was also demonstrated that sub-clustering enabled by GOMCL-sub was able to provide additional insight of selected clusters produced by GOMCL to guide further investigation.

GOMCL can be used for batch processing of multiple enrichment test results defined by the user. It is applicable for any research project where lists of genes of interest are generated. It is compatible with a wide variety of GO enrichment analysis tools publicly available, which would reduce intermediate steps needed to convert different input formats to conform to GOMCL requirements.

GOMCL currently uses the MCL algorithm for cluster identification and is compatible with commonly-used GO enrichment tools. For future versions, we consider implementing additional clustering methods, improving labeling of nodes, and supporting more GO enrichment analysis tools.

\section{Conclusion}

Lists of overrepresented GO functions from GO enrichment analyses are often long and redundant. We present GOMCL as a convenient toolkit to identify functional clusters among GO term similarity networks and further separate the resulting clusters into more informative sub-groups. It enables the user to effectively summarize

Table 1 Feature comparison of GOMCL and other tools for clustering of GO terms

\begin{tabular}{llllll}
\hline & GOMCL & Enrichment Map [10] & ClueGO [12] & DAVID [25] & POSOC [26] \\
\hline Clustering & Yes & Yes & Yes & Yes & Yes \\
Clustering basis & Jaccard Coefficient/Overlap & Jaccard Coefficient/Overlap & Kappa & Kappa & GO hierarchical \\
& Coefficient & Coefficient & statistics & statistics & distance \\
Clustering method & $\begin{array}{l}\text { Markov Clustering (MCL) } \\
\text { algorithm }\end{array}$ & Visual identification & Iterative & Iterative & Ranking \\
Sub-clustering & Yes & No & merging & No & No \\
Type of visualizationg support & Network and Hierarchy & Network & Network & Table & Table \\
$\begin{array}{l}\text { Compatible with other } \\
\text { enrichment tools }\end{array}$ & Yes & Yes & No & No & No \\
Batch processing & Yes & No & No & No & No
\end{tabular}


long lists of GO functions into biologically informative non-redundant clusters without hand-picked selections and look for major functional themes associated with the experiments. GOMCL assists with the unmet yet increasing need for interpreting large gene sets often produced from -omics studies.

\section{Supplementary information}

Supplementary information accompanies this paper at https://doi.org/10. 1186/s12859-020-3447-4

Additional file 1. GOMCL cluster information for each GO term.

Additional file 2. Similarity connections of GOMCL clusters and hierarchical connections of GOMCL cluster C1.

Additional file 3. $\mathrm{GO}$ hierarchical structure produced using $\mathrm{GOMCL}$ for cluster C2 described in Fig. 1

Additional file 4. Summary for GOMCL-sub clustering results of $\mathrm{C} 1$ described in Fig. 1.

Additional file 5. GOMCL-sub sub-cluster information for each $\mathrm{GO}$ term in cluster $\mathrm{C} 1$ described in Fig. 1.

\section{Abbreviations}

GO: Gene Ontology; MCL: Markov Clustering; JC: Jaccard Coefficient; OC: Overlap Coefficient

\section{Acknowledgments}

The authors thank High Performance Computing at Louisiana State

University (HPC@LSU) for providing computer resources to develop this tool.

\section{Availability and requirements}

Project name: GOMCL

Project home page: https://github.com/Guannan-Wang/GOMCL and www. Isugenomics.org

Operating system(s): Unix-based operating system

Programming language: Bash, Python 2.7

Other requirements: $\mathrm{MCL}$

License: GNU General Public License

Any restrictions to use by non-academics: None

\section{Authors' contributions}

GW, DHO and MD conceived the project. GW designed and implemented the code. GW and DHO tested the pipeline. GW, DHO and MD wrote the manuscript. All authors read and approved the final manuscript.

\section{Funding}

This work was supported by the National Science Foundation awards MCB 1616827, NSF-IOS-EDGE 1923589 and the Next-Generation BioGreen21 Program of Republic of Korea (PJ01317301). GW was supported by an Economic Development Assistantship award made to MD by the Louisiana State University. The funding bodies played no role in the design of the study and collection, analysis, and interpretation of data and in writing the manuscript.

\section{Availability of data and materials}

The source code of GOMCL is freely available at https://github.com/ Guannan-Wang/GOMCL and www.Isugenomics.org. The code is provided with a detailed manual and a sample test dataset used in the current study. Installation guidelines for GOMCL and associated dependencies and detailed explanations for parameters in GOMCL are available on the GitHub page.

\section{Ethics approval and consent to participate} Not Applicable.

\section{Consent for publication}

Not Applicable.

\section{Competing interests}

The authors declare that they have no competing interests.

Received: 15 October 2019 Accepted: 6 March 2020

Published online: 10 April 2020

\section{References}

1. Ogata H, Goto S, Sato K, Fujibuchi W, Bono H, Kanehisa M. KEGG: Kyoto Encyclopedia of Genes and Genomes. Nucleic Acids Res. 1999;27:29-34. https://doi.org/10.1093/nar/27.1.29.

2. Kanehisa M, Sato Y, Kawashima M, Furumichi M, Tanabe M. KEGG as a reference resource for gene and protein annotation. Nucleic Acids Res. 2016:44:D457-62. https://doi.org/10.1093/nar/gkv1070.

3. Kanehisa M, Furumichi M, Tanabe M, Sato Y, Morishima K. KEGG: new perspectives on genomes, pathways, diseases and drugs. Nucleic Acids Res. 2017;45:D353-61. https://doi.org/10.1093/nar/gkw1092.

4. Fabregat A, Jupe S, Matthews L, Sidiropoulos K, Gillespie M, Garapati P, et al. The Reactome Pathway Knowledgebase. Nucleic Acids Res. 2018;46:D64955. https://doi.org/10.1093/nar/gkx1132.

5. Cerami EG, Gross BE, Demir E, Rodchenkov I, Babur O, Anwar N, et al. Pathway Commons, a web resource for biological pathway data. Nucleic Acids Res. 2011;39(Database):D685-90. https://doi.org/10.1093/nar/gkq1039.

6. Khatri P, Sirota M, Butte AJ. Ten Years of Pathway Analysis: Current Approaches and Outstanding Challenges. PLoS Comput Biol. 2012;8: e1002375. https://doi.org/10.1371/journal.pcbi.1002375

7. Wadi L, Meyer M, Weiser J, Stein LD, Reimand J. Impact of outdated gene annotations on pathway enrichment analysis. Nat Methods. 2016;13:705-6. https://doi.org/10.1038/nmeth.3963.

8. Ashburner M, Ball CA, Blake JA, Botstein D, Butler H, Cherry JM, et al. Gene Ontology: tool for the unification of biology. Nat Genet. 2000;25:25-9. https://doi.org/10.1038/75556.

9. Carbon S, Douglass E, Dunn N, Good B, Harris NL, Lewis SE, et al. The Gene Ontology Resource: 20 years and still GOing strong. Nucleic Acids Res. 2019; 47:D330-8. https://doi.org/10.1093/nar/gky1055.

10. Merico D, Isserlin R, Stueker O, Emili A, Bader GD. Enrichment Map: A Network-Based Method for Gene-Set Enrichment Visualization and Interpretation. PLoS One. 2010;5:e13984. https://doi.org/10.1371/journal. pone.0013984.

11. Reimand J, Isserlin R, Voisin V, Kucera M, Tannus-Lopes C, Rostamianfar A, et al. Pathway enrichment analysis and visualization of omics data using g: Profiler, GSEA, Cytoscape and EnrichmentMap. Nat Protoc. 2019;14:482-517. https://doi.org/10.1038/s41596-018-0103-9.

12. Bindea G, Mlecnik B, Hackl H, Charoentong P, Tosolini M, Kirilovsky A, et al. ClueGO: a Cytoscape plug-in to decipher functionally grouped gene ontology and pathway annotation networks. Bioinformatics. 2009;25:1091-3. https://doi.org/10.1093/bioinformatics/btp101.

13. Van Dongen S. Graph Clustering by Flow Simulation. PhD thesis: University of Utrecht; 2000

14. Van Dongen S. Graph Clustering Via a Discrete Uncoupling Process. SIAM J Matrix Anal Appl. 2008:30:121-41. https://doi.org/10.1137/040608635.

15. Van Dongen S, Abreu-Goodger C. Using MCL to Extract Clusters from Networks. Methods Mol Biol. 2012:804:281-95. https://doi.org/10.1007/9781-61779-361-5_15

16. Maere S, Heymans K, Kuiper M. BiNGO: a Cytoscape plugin to assess overrepresentation of Gene Ontology categories in Biological Networks. Bioinformatics. 2005:21:3448-9. https://doi.org/10.1093/bioinformatics/ bti551.

17. Eden E, Navon R, Steinfeld I, Lipson D, Yakhini Z. GOrilla: a tool for discovery and visualization of enriched $\mathrm{GO}$ terms in ranked gene lists. BMC Bioinformatics. 2009:10:48. https://doi.org/10.1186/1471-2105-10-48.

18. Raudvere U, Kolberg L, Kuzmin I, Arak T, Adler P, Peterson H, et al. g:Profiler: a web server for functional enrichment analysis and conversions of gene lists (2019 update). Nucleic Acids Res. 2019;47:W191-8. https://doi.org/10. 1093/nar/gkz369.

19. Tian T, Liu Y, Yan H, You Q, Yi X, Du Z, et al. agriGO v2.0: a GO analysis toolkit for the agricultural community, 2017 update. Nucleic Acids Res. 2017; 45:W122-9. https://doi.org/10.1093/nar/gkx382.

20. Klopfenstein DV, Zhang L, Pedersen BS, Ramírez F, Warwick Vesztrocy A, Naldi A, et al. GOATOOLS: A Python library for Gene Ontology analyses. Sci Rep. 2018;8:10872. https://doi.org/10.1038/s41598-018-28948-z. 
21. Shannon P. Cytoscape: A Software Environment for Integrated Models of Biomolecular Interaction Networks. Genome Res. 2003;13:2498-504. https:// doi.org/10.1101/gr.1239303.

22. Wendrich JR, Möller BK, Li S, Saiga S, Sozzani R, Benfey PN, et al. Framework for gradual progression of cell ontogeny in the Arabidopsis root meristem. Proc Natl Acad Sci. 2017;114:E8922-9. https://doi.org/10.1073/pnas. 1707400114

23. Foroozani M, Zahraeifard S, Oh D-H, Wang G, Dassanayake M, Smith AP. Low-Phosphate Chromatin Dynamics Predict a Cell Wall Remodeling Network in Rice Shoots. Plant Physiol. 2020;182:1494-509. https://doi.org/10. 1104/pp.19.01153.

24. Gene Ontology Consortium. The Gene Ontology (GO) database and informatics resource. Nucleic Acids Res. 2004;32:258D-261. https://doi.org/ 10.1093/nar/gkh036.

25. Huang DW, Sherman BT, Lempicki RA. Systematic and integrative analysis of large gene lists using DAVID bioinformatics resources. Nat Protoc. 2009;4: 44-57. https://doi.org/10.1038/nprot.2008.211.

26. Joslyn CA, Mniszewski SM, Fulmer A, Heaton G. The Gene Ontology Categorizer. Bioinformatics. 2004;20(Suppl 1):i169-77. https://doi.org/10. 1093/bioinformatics/bth921.

\section{Publisher's Note}

Springer Nature remains neutral with regard to jurisdictional claims in published maps and institutional affiliations.

Ready to submit your research? Choose BMC and benefit from:

- fast, convenient online submission

- thorough peer review by experienced researchers in your field

- rapid publication on acceptance

- support for research data, including large and complex data types

- gold Open Access which fosters wider collaboration and increased citations

- maximum visibility for your research: over $100 \mathrm{M}$ website views per year

At $\mathrm{BMC}$, research is always in progress.

Learn more biomedcentral.com/submissions 